\title{
Particle acceleration and reconnection in the solar wind
}

G. P. Zank, P. Hunana, P. Mostafavi, J. A. le Roux, G. M. Webb, O. Khabarova, A. C. Cummings, E. C. Stone, and R. B. Decker

Citation: AIP Conference Proceedings 1720, 070011 (2016); doi: 10.1063/1.4943848

View online: http://dx.doi.org/10.1063/1.4943848

View Table of Contents: http://aip.scitation.org/toc/apc/1720/1

Published by the American Institute of Physics 


\title{
Particle Acceleration and Reconnection in the Solar Wind
}

\author{
G. P. Zank ${ }^{1,2}$, P. Hunana ${ }^{1,2}$, P. Mostafavi ${ }^{1,2}$, J. A. le Roux ${ }^{1,2}$, G. M. Webb ${ }^{1,2}$, O. \\ Khabarova $^{3}$, A.C. Cummings ${ }^{4}$, E.C. Stone ${ }^{4}$ and R.B. Decker ${ }^{5}$ \\ ${ }^{1}$ Center for Space Plasma and Aeronomic Research (CSPAR), University of Alabama, Huntsville, AL 35805, USA \\ ${ }^{2}$ Department of Space Science, University of Alabama, Huntsville, AL 35899, USA \\ ${ }^{3}$ Heliophysical Laboratory, IZMIRAN, Troitsk, Moscow 142190, Russia \\ ${ }^{4}$ California Institute of Technology, Mail Code 290-17, Pasadena, CA 91125, USA \\ ${ }^{5}$ Johns Hopkins University/Applied Physics Lab., Laurel, MD 20723-6099, USA
}

\begin{abstract}
An emerging paradigm for the dissipation of magnetic turbulence in the supersonic solar wind is via localized quasi2D small-scale magnetic island reconnection processes. An advection-diffusion transport equation for a nearly isotropic particle distribution describes particle transport and energization in a region of interacting magnetic islands $[1 ; 2]$. The dominant charged particle energization processes are 1) the electric field induced by quasi-2D magnetic island merging, and 2) magnetic island contraction. The acceleration of charged particles in a "sea of magnetic islands" in a super-Alfvénic flow, and the energization of particles by combined diffusive shock acceleration (DSA) and downstream magnetic island reconnection processes are discussed.
\end{abstract}

\section{Introduction}

Reconnection associated with merging and contracting plasmoids (flux ropes) can lead to the first-order Fermi energization of electrons and ions trapped in the islands [1-6]. Rather hard power law spectra are often found in simulations [6; 7]. The transport theory developed by Zank et al. [1] and le Roux et al. [2] incorporates energy changes to the charged particle distribution function by magnetic island contraction and merging.

In situ evidence that electrons and possibly ions are energized in solar wind regions filled with magnetic islands or plasmoids has emerged in the past few years (see $[1 ; 8]$ for reviews). Possible evidence of electron acceleration by magnetic islands may have been found by Wang et al. [9]. Quiet time superhalo ( $2-20 \mathrm{keV})$ electrons were observed i) almost exclusively in the slow solar wind, ii) to be isotropically distributed, and iii) to possess power law spectra in particle speed $c^{-\gamma}$, with $\gamma \in[5,8.7]$. Nearly half the spectra lie in the interval $\gamma \in[6.5,7.5]$. We have suggested that charged particles can be accelerated during quiet times in the vicinity of the heliospheric current sheet $[1 ; 8 ; 10]$, where a large population of magnetic islands may be expected. Power law distributions for accelerated electrons with spectral indices ranging from $\sim 6-7$ between $\sim 0.8-2$ AU for the simplest case of magnetic island merging can be derived from the theory of [1]. The origin of the quiet time electron superhalo spectra [9] may be due to stochastic particle acceleration by distributed magnetic islands in the neighborhood of the heliospheric current sheet.

\section{Charged particle energization, magnetic islands, and transport formalism}

Three processes can increase the energy of test particles interacting with a dynamical "sea" of magnetic islands. (1) As an elongated island contracts, trapped particles experience repeated reflections at either end of the contracting plasmoid. Particles are energized by a first-order Fermi process [3] due to curvature drift in the direction of the induced electric field generated at the strongly curved magnetic field at the endpoints of a contracting flux rope, or in the outflow regions of reconnection sites between merging plasmoids $[1-3 ; 11 ; 12]$. (2) Depending on whether the magnetic island contraction is compressible or incompressible, the magnetic field strength will either increase or decrease. In the former case, betatron acceleration of the particle will contribute to the energy gain, whereas in the latter case, betatron deceleration will result, both a consequence of charged particle conservation of magnetic moment $[1 ; 2]$. The compressible plasmoid contraction case yields a first-order Fermi energization mechanism [1;2], whereas the incompressible mechanism corresponds to a second-order Fermi energization mechanism $[1 ; 2 ; 5]$. (3) 
Particles trapped inside an island merger will repeatedly interact with the "anti-reconnection" electric field induced by the merging process $[6 ; 13 ; 14]$, leading to electron heating and energization. Some test particle simulations [6;14] concluded that particle acceleration by the induced electric field associated with small-scale magnetic island merging may be the dominant energization process for particles in reconnection layers. However, test particle simulations of electron acceleration in a region of cascading magnetically reconnecting magnetic islands [12] suggest that electron energization is due primarily to magnetic field curvature and gradients, i.e., by either compressible or incompressible magnetic island contraction. These two sets of somewhat contradictory simulations do not make it clear which process is likely to dominate the energization of charged particles in flux rope-related reconnection processes. Accordingly, we retain both mechanisms in our transport theory $[1 ; 2]$ and explore the effects of both.

Zank et al. [1] derived a transport equation for a gyrotropic distribution of particles experiencing pitch-angle scattering and energization via the three reconnection-related processes described above for a super-Alfvénic plasma flow. The gyrophase-averaged or focused transport equation can be simplified further by assuming a nearly isotropic particle distribution, yielding a first-order correct advection-diffusion transport equation [1],

$$
\frac{\partial f}{\partial t}+\left(U_{i}+3\left|V_{E}\right| b_{i}\right) \frac{\partial f}{\partial x_{i}}-\frac{c}{3} \frac{\partial U_{i}}{\partial x_{i}} \frac{\partial f}{\partial c}+\frac{1}{c^{2}} \frac{\partial}{\partial c}\left(\frac{c^{3}}{3} 2 \eta_{c} f\right)=\frac{\partial}{\partial x_{i}}\left(K_{i j} \frac{\partial f}{\partial x_{j}}\right)-c \frac{\partial}{\partial x_{i}}\left(\left|V_{E}\right|_{i} \frac{\partial f}{\partial c}\right)-\left|V_{E}\right|_{i} c \frac{\partial^{2} f}{\partial x_{i} \partial c},
$$

where $f=f(\mathbf{x}, t, c)$ is a charged particle distribution function of particle position $\mathbf{x}$, time $t$, and non-relativistic speed $c$. $\mathbf{U}$ is the large-scale plasma flow velocity, $\mathbf{b} \equiv \mathbf{B} /|\mathbf{B}|$ the unit vector in the direction of the magnetic field, $\left|V_{E}\right| \equiv 1 / 3 q / m \delta E_{3} \tau_{s}$ relates the anti-reconnection electric field along the $\mathbf{b}$ direction $\delta E_{3}$ to particle scattering via $\tau_{s}$, the characteristic pitch-angle scattering time, and $q$ and $m$ are the particle charge and mass respectively. $\eta_{c}$ describes the characteristic contraction rate of magnetic islands $[1 ; 2 ; 5 ; 11 ; 12]$, and $\mathbf{K}$ describes the spatial diffusion of the nearly isotropic distribution of particles due to pitch-angle scattering. Three energization terms are present in (1), these being (i) the divergence of the large-scale background flow velocity; (ii) a similar term for microscopic magnetic island contraction, and (iii) two mixed derivative terms with coefficient $\left|V_{E}\right|$ that incorporate the anti-reconnection electric field.

The phase space conservation form of the transport equation (1) is given by

$$
\frac{\partial f}{\partial t}+\frac{\partial S_{i}}{\partial x_{i}}+\frac{1}{c^{2}} \frac{\partial}{\partial c}\left(c^{2} J_{p}\right)=0
$$

where $\mathbf{S} \equiv-\mathbf{K} \cdot \nabla f-(c / 3)(\partial f / \partial c)\left(\mathbf{U}-3\left|\mathbf{V}_{E}\right|\right)$ and $J_{p} \equiv(c / 3)\left(\mathbf{U}+\left|\mathbf{V}_{E}\right|\right) \cdot \nabla f+(2 c / 3) \eta_{c} f$ denote streaming in space and momentum space respectively. Equation (2) determines the particle boundary conditions at a shock wave.

\section{Charged particle acceleration in a super-Alfvénic flow}

Consider perhaps the simplest of problems: a $1 \mathrm{D}$ incompressible super-Alfvénic flow, $\nabla \cdot \mathbf{U}=0$, with a steady injection of particles of fixed initial speed $c_{0}$ at the origin. Further assume that the spatial diffusion coefficient is constant. The steady-state $1 \mathrm{D}$ equation may be expressed as $\left(\xi \equiv \ln \left(c / c_{0}\right), \kappa=K_{x x}\right.$, and $Q \equiv n_{0} c_{0} /\left(4 \pi c^{2}\right)$, and $n_{0}$ the injected number density)

$$
\frac{\partial^{2} f}{\partial x^{2}}-2 \frac{\left|V_{E}\right|}{\kappa} \frac{\partial^{2} f}{\partial x \partial \xi}-\frac{U+3\left|V_{E}\right|}{\kappa} \frac{\partial f}{\partial x}-\frac{2 \eta_{c}}{3 \kappa} \frac{\partial f}{\partial \xi}-\frac{2 \eta_{c}}{\kappa} f=-\frac{Q}{\kappa} \delta\left(c-c_{0}\right) \delta(x),
$$

which is hyperbolic. The solution can be expressed in terms of characteristic diffusion $\tau_{d i f f}$, island contraction $\tau_{c}$, and diffusive length $L_{d i f f}$ scales, and the Alfvén Mach number $M_{A}[1]$. Hence

$$
\begin{gathered}
f\left(x, c / c_{0}\right)=\frac{n_{0}}{8 \pi c_{0}^{2} V_{A}}\left(\frac{c}{c_{0}}\right)^{-w} \exp \left[-\frac{2 \tau_{d i f f}}{3 \tau_{c}} M_{A} \frac{x}{L_{d i f f}}\right] I_{0}(\Phi) H\left[\ln \left(c / c_{0}\right)\right] H\left[\ln \left(c / c_{0}\right)+\frac{2}{M_{A}} \frac{x}{L_{d i f f}}\right] ; \\
w \equiv\left(3+M_{A}+2 \tau_{d i f f} /\left(3 \tau_{c}\right) M_{A}^{2}\right) / 2 ; \quad \Phi \equiv \sqrt{\frac{\tau_{d i f f}}{3 \tau_{c}} M_{A}^{2}\left(M_{A}-3+\frac{\tau_{d i f f}}{3 \tau_{c}} M_{A}^{2}\right)}\left(\left(\ln \left(c / c_{0}\right)^{2}+\frac{4}{M_{A}} \ln \left(c / c_{0}\right) \frac{x}{L_{d i f f}}\right)^{1 / 2} .\right.
\end{gathered}
$$

Solutions (4) i.e., for $c>c_{0}$, are plotted in Fig. 1. The solutions are power laws for virtually all values of $c>c_{0}$ and the slope depends on the choice of $M_{A}$ and $\tau_{c} / \tau_{\text {diff }}$, and is determined by the modified Bessel function. 

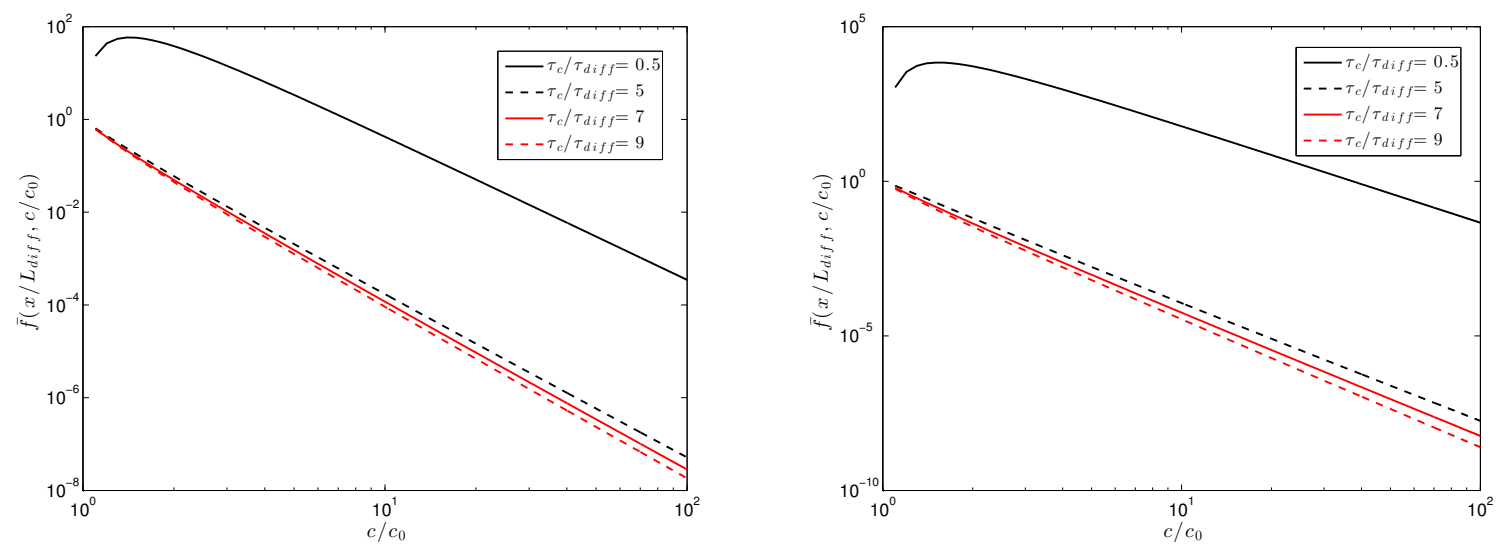

FIGURE 1. Plot of the normalized solution (4) for particles accelerated by the anti-reconnection electric field and magnetic island contraction. Four curves are plotted, one for values of $\tau_{c} / \tau_{\text {diff }}<1$ and three for $\tau_{c} / \tau_{\text {diff }}>1$. The parameter $\left|V_{E}\right|$ is related to $M_{A}$ [1]. (Left) These solutions assume that $M_{A}=7$, possibly appropriate to the inner heliosphere $(<1 \mathrm{AU})$ or during solar minimum. (Right) These solutions assume that $M_{A}=11$, possibly appropriate to the outer heliosphere ( $\left.>\sim 2 \mathrm{AU}\right)$ or during solar maximum.

\section{Combined DSA and downstream magnetic island acceleration}

Equation (1) is valid for super-Alfvénic flows. Since the flow downstream of a fast-mode shock is super-Alfvénic, the extended transport equations (1) and (2) can be used at fast-mode shocks. In the context of the shock evolutionary conditions, upstream perturbations incident on a shock generate vortical fluctuations $[15 ; 16]$ that are advected away from the shock downstream. We adopt a planar shock geometry, and solve a spatially $1 \mathrm{D}$ form of the transport equation (1) for prescribed constant upstream and downstream flows $U_{1}$ and $U_{2}$. The normalized solution is (see details in [17])

$$
\begin{aligned}
\bar{f}(\bar{x}, \xi) & =\bar{g}(\xi) e^{r \bar{x}}, \quad \bar{x}<0 \\
\bar{f}(\bar{x}, \xi) & =\exp \left[-\frac{\tau_{d i f f} / \tau_{c}}{3} \frac{U_{2}}{\left|V_{E}\right|} \bar{x}\right] \bar{g}(\xi)+\frac{\left|V_{E}\right|}{U_{2}} \bar{x} \exp \left[-\frac{\tau_{d i f f} / \tau_{c}}{3} \frac{U_{2}}{\left|V_{E}\right|} \bar{x}\right] \\
& \times \int_{0}^{\xi} \bar{g}(\tau) e^{s_{1}(\xi-\tau)} \frac{\beta I_{1}\left(\beta \sqrt{(\xi-\tau)\left(\xi-\tau+2\left(\left|V_{E}\right| / U_{2}\right) \bar{x}\right)}\right)}{\sqrt{(\xi-\tau)\left(\xi-\tau+2\left(\left|V_{E}\right| / U_{2}\right) \bar{x}\right)}} d \tau, \quad \bar{x}>0 ; \\
\bar{g}(\xi) & =\frac{1}{r-1} \frac{1}{\Gamma} \frac{1}{\alpha-3}\left[\left((a-3)+d\left(s_{1}+3\right)\right) e^{-3 \xi}+\left((\alpha-a)-d\left(s_{1}+\alpha\right)\right) e^{-\alpha \xi}\right. \\
& \left.+d \beta \int_{0}^{\xi}\left(e^{-3 \tau}-e^{-\alpha \tau}\right) \frac{e^{s_{1}(\xi-\tau)}}{\xi-\tau} I_{1}(\beta(\xi-\tau)) d \tau\right] .
\end{aligned}
$$

Solutions (5) and (6) yield power law accelerated particle spectra at the shock. Two predictions can be made for the general coupled DSA-reconnection particle acceleration model, both of which distinguish this process from conventional DSA. For a sufficiently large value of the downstream parameter $U_{2} /\left|V_{E}\right|$, the particle intensity profile peaks downstream of the shock and the peak occurs further downstream of the shock with increasing charged particle energy.

We test the above predictions using the Voyager 2 (V2) CRS [18] observations of cosmic rays immediately upstream and downstream of the heliospheric termination shock (HTS). In Figure 2, we plot 13-day moving averages with 1 day resolution of the proton flux of a particular energy normalized to its value at the time of the shock crossing. The 10 energy bins, correspond to an energy range of $[1.8,22] \mathrm{MeV}$. Several key points are apparent. The first is that an exponential-like increase in the particle intensity immediately ahead of the shock is present for all energies. This is consistent with DSA only and no additional acceleration processes ahead of the HTS are necessary. However, the downstream normalized intensities are completely different from the predictions of conventional DSA theory, which predicts a flat normalized intensity profile equal to 1 for all energies. Instead, each energy is amplified above its value at the HTS and the amplification factor is very clearly ordered by increasing energy. The location of the particle 
intensity peak appears to increase with increasing energy. In Figure 2, we plot a set of normalized particle intensity solutions for the general case in the same format as the observations (see [17] for relevant parameters). The theoretical curves show that for the corresponding set of normalized energies, we obtain an amplification factor that increases with increasing energy, and for which the amplification factor is roughly consistent across the observed energies. Moreover, the location of the intensity peak increases with increasing energy. Based on the observations illustrated
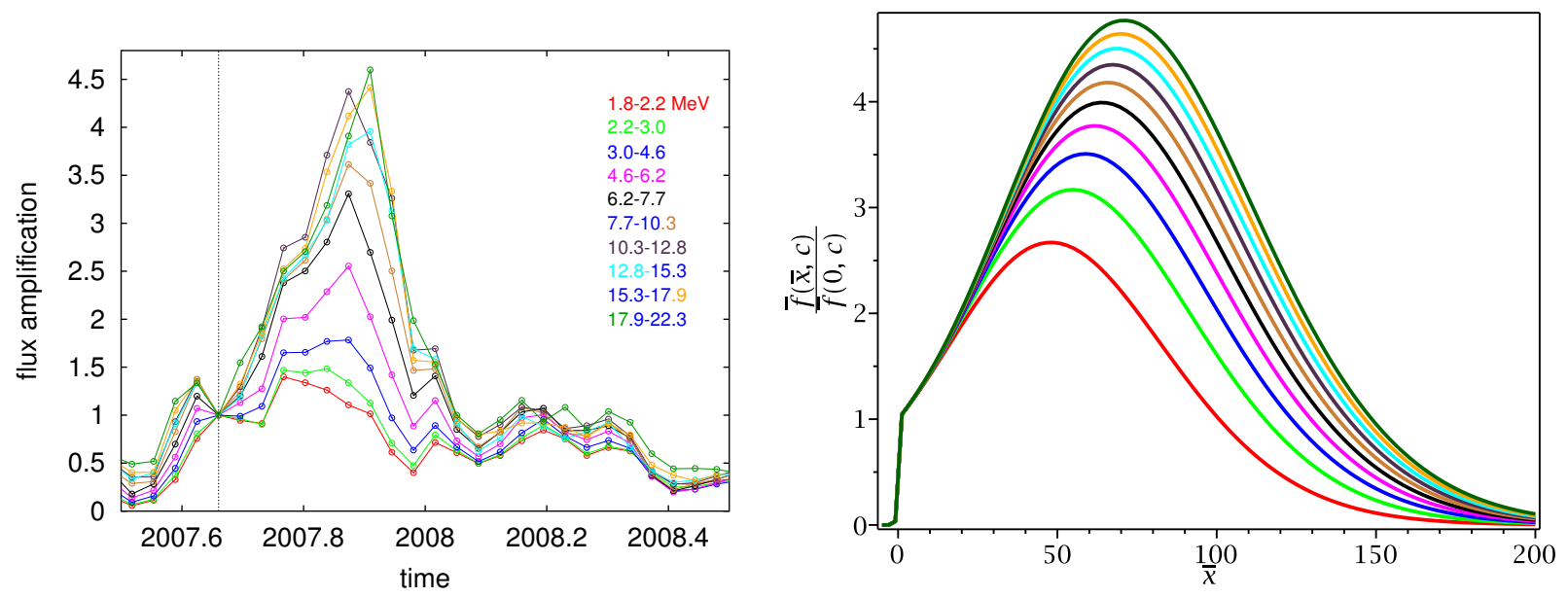

FIGURE 2. (Left) Flux amplification factor obtained from V2 proton data for the 10 energy ranges listed in the figures, measured in MeV. (Right) The amplification factor $f\left(x, c / c_{0}\right) / f\left(0, c / c_{0}\right)$ for fixed energies as a function of position for the general case.

in Figure 2, it appears that the DSA-magnetic island acceleration mechanism provides an explanation for the overall intensity profile observed by V2, both upstream and downstream of the HTS. The parameters that are consistent with the V2 observations suggest that the dominant downstream energization process is magnetic island contraction.

Acknowledgments We acknowledge partial support from NASA grants NNX08AJ33G, Subaward 37102-2, NNX14AC08G, NNX14AJ53G, RR185-447/4944336 and NNX12AB30G, NNX14AF43G, NNX15AI65.

\section{REFERENCES}

[1] Zank G P, le Roux J A, Webb G M, Dosch A and Khabarova O 2014 Ap. J. 79728

[2] le Roux J A, Zank G P, Webb G M and Khabarova O 2015 Ap. J. 801112

[3] Drake J F, Swisdak M, Che H and Shay M A 2006 Nature 443 553-556

[4] Drake J F, Swisdak M, Schoeffler K M, Rogers B N and Kobayashi S 2006 Geophys. Res. Lett. 33 L13105

[5] Drake J F, Swisdak M and Fermo R 2013 Ap. J. Lett. 763 L5 (Preprint 1210.4830)

[6] Oka M, Phan T D, Krucker S, Fujimoto M and Shinohara I 2010 Ap. J. 714 915-926 (Preprint 1004.1154)

[7] Guo F, Liu Y H, Daughton W and Li H 2015 ArXiv e-prints (Preprint 1504.02193)

[8] Khabarova O, Zank G P, Li G, le Roux J A, Webb G M, Dosch A and Malandraki O E 2015 Ap. J. 80824 (Preprint 1504.06616)

[9] Wang L, Lin R P, Salem C, Pulupa M, Larson D E, Yoon P H and Luhmann J G 2012 Ap. J. Lett. 753 L23

[10] Zank G P, Hunana P, Mostafavi P, le Roux J A, Li G, Webb G M and Khabarova O 2015 Journal of Physics Conference Series 642012031

[11] Bian N H and Kontar E P 2013 Physical Review Letters 110151101 (Preprint 1302.6090)

[12] Zhou X, Büchner J, Bárta M, Gan W and Liu S 2015 Ap. J. 8156 (Preprint 1504.06486)

[13] Pritchett P L 2008 Physics of Plasmas 15102105

[14] Le A, Karimabadi H, Egedal J, Roytershteyn V and Daughton W 2012 Physics of Plasmas 19072120

[15] McKenzie J F and Westphal K O 1968 Physics of Fluids 11 2350-2362

[16] Giacalone J and Jokipii J R 2007 Ap. J. Lett. 663 L41-L44

[17] Zank G P, Hunana P, Mostafavi P, Le Roux J A, Li G, Webb G M, Khabarova O, Cummings A, Stone E and Decker R 2015 Ap. J. 814137

[18] Stone E C, Vogt R E, McDonald F B, Teegarden B J, Trainor J H, Jokipii J R and Webber W R 1977 Space Sci. Rev. $21355-376$ 